\title{
A cadaveric case report of dual thyroid: ectopic and normal location thyroid with brief review of literature
}

\begin{abstract}
Ectopic thyroid tissue is a rare congenital anomaly due to developmental defect in thyroid gland. Ectopic, accessory, or aberrant thyroid refers to the presence of thyroid tissue in locations other than normal pretracheal position in the anterior neck region. The thyroid dysgenesis occurs due to abnormal organogenesis of the thyroid gland leading to morphological variations, such as hypoplasia, hemiagenesis, and agenesis with ectopic thyroid being the most common form of thyroid dysgenesis. Here, we are presenting a case of dual thyroid in the form of ectopic or accessory thyroid tissue along with normal location thyroid found during a routine dissection of a female cadaveric body. The ectopic thyroid tissue was seen as an oval structure about 2.2 $\mathrm{cm} \times 1.6 \mathrm{~cm} \times 1.0 \mathrm{~cm}$, situated in the midline near the hyoid bone. It was located $10.2 \mathrm{~cm}$ superior to a normally located thyroid gland and consistent with the track of the thyroglossal duct. The ectopic thyroid tissue could be asymptomatic or it could present with any disorder affecting the main thyroid gland including malignancy. The significance of this cadaveric case report and a brief review of the literature on normal location thyroid gland and ectopic thyroid highlight an uncommon but potentially important congenital anomaly that can be a source of diagnostic confusion and a challenge for therapeutic management.
\end{abstract}

Keywords: Thyroid gland, Ectopic, Accessory, Aberrant, Dysgenesis, Congenital Anomaly
Volume 6 Issue 3 - 2018

\author{
Devendra A Sawant, 'Thomas F Moore, ${ }^{2}$ Alan \\ W Cashell' \\ 'School of Anatomical Science, College of Medical Science, \\ Alderson Broaddus University, Philippi,WV, USA \\ ${ }^{2}$ School of Physician Assistant Studies, College of Medical \\ Science, Alderson Broaddus University, Philippi,WV, USA
}

\begin{abstract}
Correspondence: Devendra A Sawant, MD, MPH, Ph.D, Chairman \& Assistant Professor, School of Anatomical Science, Alderson Broaddus University College of Medical Science, 101 College Hill Drive, Philippi, West Virginia, 26416 USA, Tel (304) 457-6587,

Email sawantda@ab.edu
\end{abstract}

Received: February 19, 2018 | Published: May 23, 2018

\section{Introduction}

The thyroid gland is a highly vascularized endocrine gland that frequently present with varied range of morphological anomalies. ${ }^{1}$ Most of these anatomical variations can be traced to an aberration during organogenesis. These structural deformities in thyroid gland due to defective organogenesis are termed as thyroid dysgenesis that includes anomalies such as ectopic thyroid, agenesis, hemiagensis, and hypoplasia. Ectopic, accessory, or aberrant thyroid refers to the thyroid tissue located in the places other than the normal pretracheal site in the anterior neck at the level of 5th cervical to 1st thoracic (C5-T1) vertebrae., ${ }^{2,3}$

The first report of lingual thyroid was presented by Hickmann in $1869 .{ }^{4}$ Subsequent descriptions with categorizations of ectopic thyroid include the works of Marshall in 1895 and Netter in 1965. Also, Bhatnagar et al in 1997 proposed to classify these morphological variations depending on the isthmus of the thyroid gland. According to their proposed classification, the first group was the normal location thyroid gland with structural abnormalities such as aplasia, sub-isthmic accessory lobe and thyroid gland with pyramidal lobe. The second group was formed as thyroid associated anomalies that included thyroglossal duct with or without nodules, separate nodules on thyrohyoid muscle or in the neck, and musculus levator glandulae thyroideaea. And the third group is referred as ectopic thyroid tissue that included thyroid tissue in abnormal location such as lingual (tongue), thorax (heart), abdominal (liver) and other rare sites. ${ }^{2,5}$

Epidemiologically, ectopic thyroid tissue is a sporadic embryological deformity that is usually noticed during autopsy and that has a prevalence of up to $10 \%$. However, in general population the prevalence of ectopic thyroid tissue is around 1 per 100,000-300,000 people, which increases to 1 per $4000-8000$ patients with thyroid disease. The incidence of thyroid ectopy is not widely reported. Ectopic thyroid has a gender predisposition with male to female ratio of 1:4., ${ }^{2,6-8}$ In this report, we present a female cadaveric case involving ectopic thyroid tissue along with a brief literature review including pertinent aspects of anatomy, embryology, histology, and physiology. We also include relevant diagnostic and therapeutic strategies. This focused review with emphasis on clinical implications could be immensely beneficial while studying thyroid gland and diagnosing thyroid disorders or carrying out surgical procedures involving thyroid.

\section{Embryological perspective}

The thyroid gland is the first endocrine gland to form during embryonic period around 24 days after fertilization. During embryological growth thyroid parenchyma develops from both endoderm and ectoderm. A median endodermal thickening in the floor of the primitive pharynx between the first and second branchial arches gives rise to an outgrowth called as the thyroid diverticulum just caudal to the future site of the tongue bud which will grow in to future tongue. The thyroid diverticulum begins to develop in to thyroid gland as it gradually descends into the neck, ventral to the developing hyoid bone and the lateral cartilages, while remaining connected to the tongue through thyroglossal duct. The opening of the thyroglossal duct in the tongue is known as foramen cecum. During organogenesis, the hollow thyroid diverticulum start becoming solid gland which then divides in to two right and left lateral lobes. Anterior to the second and third tracheal rings the lateral lobes are interconnected with an isthmus in between. The thyroid gland reaches its normal destination in the neck along with its final characteristic shape around 7 week of embryonic development. By this time, the thyroglossal duct has normally degenerated and disappeared except 
foramen cecum that remain as a small blind pit on the tongue. The ectodermal component of thyroid parenchyma originate from the neuroectodermal ultimobranchial body giving rise to parafollicular or $\mathrm{C}$ (clear) cells of the thyroid gland, whereas endodermal follicular cells are developed from thyroid anlage. . $^{3,4,9,10}$

The thyroid anlage is a small thickening of the endodermal epithelium in the foregut consisting of endodermal cells. This unique region is situated in the midline on the posterior part of the embryonic mouth cavity. In the stages of morphogenesis, the thyroid anlage appears at embryonic day E20-E22, the thyroid bud migration begins E24, and the thyroglossal duct disappears around E30-E40. The precursors of parafollicular cells or $\mathrm{C}$ cell of neural crest origin migrate to a paired structure called as ultimobranchial bodies placed on the sides of the developing neck. The ultimobranchial body is embryologically derived from the fourth pharyngeal pouch. This process of thyroid migration is completed at embryonic day E45-E50. The thyroid anlage and the ultimobranchial bodies ultimately merge to form thyroid gland. The cells originating from the thyroid anlage becomes thyroid follicular cells and that collectively organizes to form the thyroid follicles. This merger with ultimobranchial bodies takes place at embryonic day E60. While the beginning of folliculogenesis occurs at embryonic day E70 with the cells originating from ultimobranchial bodies get placed in interfollicular space forming parafollicular or $\mathrm{C}$ cells of the adult thyroid gland. ${ }^{2,3}$

\section{Histological perspective}

The thyroid gland has a thin covering of the connective tissue layer called as capsule that divides the glandular lobe in to lobules for varied size and shapes. Each lobe of the thyroid gland is made up of numerous follicles. These follicles form the functional units of thyroid gland. Each follicle is $200-300 \mu \mathrm{m}$ in diameter lined by a single layer of follicular cells originated from endodermal thyroid anlage. These follicular cells could be cuboidal to low columnar epithelial cells that stain slightly basophilic. They have spherical nuclei and one or more prominent nucleoli. The basal surface of the follicular cells is supported by basal lamina. The apical surface faces the lumen of the follicles which is filled with a gelatinous substance referred to as colloid. The colloid stains with both eosinophilic and basophilic dyes and is periodic acid-Schiff (PAS) positive. The colloid in the follicular cells is made up of glycoprotein called thyroglobulin that undergoes iodination to synthesize thyroid hormone. Thyroglobulin is primarily an inactive form of the thyroid hormones T3 and T4. Stimulated by thyroid stimulating hormone (TSH), the colloid is resorbed by the follicular cells to form colloidal resorption droplets. Within the cells, it is degraded to release T3 and T4. Electron microscopy demonstrates a Golgi apparatus within the follicular cells in a supranuclear position. The rough endoplasmic reticulum (RER) is present at the apical surface of the cells that have a role in the manufacturing of hormones. The lysosomes seen by electron microscopy may be visible as PAS positive or lipid droplets. The shape of follicular cells depends upon their activity level which is regulated by TSH. The resting follicular cells are large squamous or cuboidal, whereas functionally active follicular cells will be columnar. These follicles are surrounded by parafollicular cells or C (clear) cells of neuroectodermal origin. The $\mathrm{C}$ cells produce calcitonin that lowers blood calcium by suppressing bone resorption by osteoclasts. These $\mathrm{C}$ cells are pale staining and difficult to appreciate by light microscopy. They are peripheral to the follicular cells and not incontact with colloid. They contain many electron dense granules and a prominent Golgi apparatus. ${ }^{3,6,10,11}$

\section{Anatomical perspective}

The thyroid gland normally weighs approximately 25 grams, but shows considerably variation in size with age, sex, race, physiological state of the body, and geographical location of the individual. It is slightly heavier in women and the weight increases further more during the state of menstruation and pregnancy. ${ }^{1,3}$ The thyroid gland is located at the level of C5-T1 vertebras anterior to the trachea and inferior to the sternothyroid and sternohyoid muscles. The thyroid gland is enclosed in its capsule surrounded by a layer of fascia which is thickened posteriorly and attached to the cricoid cartilage and upper tracheal rings known as Suspensory ligament of Berry. The thyroid gland getting wrapped around by this pretracheal fascia makes the gland mobile during deglutition. Thyroid gland consists of two lobes on both right and left sides with an isthmus attaching the two lateral lobes in the center. The diameter of the thyroid lobes antero-posteriorly is $10-20 \mathrm{~mm}$ and medio-laterally is $10-30 \mathrm{~mm}$ with the isthmus around $5 \mathrm{~mm}$ in thickness. In nearly half of the population, thyroid glands have a third lobe shaped in a pyramidal form. The pyramidal lobe that varies in size originates from the isthmus and extends till upper segment of the lamina of the thyroid cartilage usually on the left side of the paramedian plane. In some instances, the pyramidal lobe may be connected superiorly to the body of the hyoid bone by a smooth muscle called the levator glandulae thyroideae.

The pyramidal lobe along with the associated smooth muscle develops from remnants of the thyroglossal duct. Another landmark in the anatomy of the thyroid gland is a thickening of thyroid parenchyma present near the posteriolateral aspect of the thyroid gland called as Tubercle of Zuckerkand., , $, 10,12-14^{2}$ The highly vascularized thyroid gland is supplied by superior and inferior thyroid artery branches of external carotid artery and thyrocervical trunk, a branch of subclavian artery respectively. In some individuals an additional thyroidea ima artery, a branch of brachiocephalic trunk or aortic arch may also supply thyroid gland. The superior thyroid artery is in close association with external laryngeal nerve, while inferior thyroid artery is in close proximity to recurrent laryngeal nerve which makes this relationship clinically important because of increase in possibility of iatrogenic nerve injury during thyroid surgical procedures. The venous drainage of thyroid gland is via superior, middle, and inferior thyroid veins. The superior and middle thyroid veins drains in internal jugular vein whereas the inferior thyroid vein drains in to left brachiocephalic vein or directly in to superior vena cava. The lymphatics from thyroid gland may drain directly in to thyroid duct without intervening lymph nodes in between. Also in some case the thyroid gland may drain in to pretracheal, paratracheal, and prelarynegeal group of lymph nodes. The innervation for thyroid gland comes from superior, middle and inferior cervical sympathetic ganglia. The post ganglionic nerve fibers from inferior cervical ganglion further communicate with external and recurrent laryngeal nerves which travel along with superior and inferior thyroid arteries. ${ }^{3,9,15}$

\section{Physiological perspective}

The thyroid hormone is secreted by the thyroid follicular cells of the thyroid gland in response to thyroid stimulating hormone (TSH) from anterior pituitary gland. The circulating thyroid hormone in a cyclical process stimulates anterior pituitary gland to secrete TSH, which in turn acts on the TSH receptor present on the basolateral membranes of thyroid follicular cells to release thyroid hormones. TSH facilitate iodide uptake by thyroid follicular cells through sodium/iodide symporter, followed by iodination of tyrosine residues 
to form monoiodotyrosines and diiodotyrosines in the glycoprotein thyroglobulin. The coupling of these mono and di iodotyrosines are required to form triiodothyronine (T3) and thyroxine (T4) hormone that remain bound to thyroglobulin within the colloid of the thyroid follicular cells. ${ }^{10,16-18}$ The T3 and T4 thyroid hormones are released in the blood stream by breaking their complex with thyroglobulin within the colloid. In the blood, most of the T3 and T4 thyroid hormones are transported by binding to serum proteins such as thyroxine binding globulin, thyroxine binding prealbumin and albumin, whereas the small amount T3 and T4 thyroid hormones which remains free in the blood circulation are biologically active form of hormones. The $\mathrm{T} 3$ hormone is more active than T4 with L-Isomers (levo isomers) being the naturally occurring forms of T3. In the peripheral tissues, $\mathrm{T} 3$ hormone is synthesized by the process of deiodination of $\mathrm{T} 4$ where one iodine molecule is removed and T4 is converted back to $\mathrm{T} 3$ hormone. The action of T3 takes place within few hours where as T4 acts slowly taking 4-14 days, however T3 is the physiologically more important hormone than T4. ${ }^{10,17,18}$ Thyroid hormone production is regulated by hypothalamo-pituitary-thyroid axis and feedback pathways (Figure 1). The thyroid stimulating hormone-releasing hormone or thyrotropin-releasing hormone (TRH) is secreted by the neurons in the paraventricular nucleus (PVN) of the hypothalamus. The TRH neurons of the median eminence of the hypothalamus, forms the part of the hypophyseal portal system, that transport thyrotropinreleasing hormone from hypothalamus to the anterior pituitary gland. TRH stimulates secretion of TSH from anterior pituitary gland which in turn stimulates thyroid hormone production from the thyroid gland. The thyroid gland releases $\mathrm{T} 4$ in the circulation, which is converted to the active form $\mathrm{T} 3$ by deiodinase enzymes. ${ }^{19}$

Thyroxine hormone (T4) that are circulating in the blood stream can have negative feedback on TRH production by their action on PVN of the hypothalamus entering through the blood-brain barrier as well as through the cerebrospinal fluid. The T4 is then carried to median eminence and arcuate nucleus by tanycytes lining the third ventricle and astrocytes. The type 2 deiodinase enzymes present in these cells of median eminence and arcuate nucleus convert T4 to active T3 hormone. In arcuate nucleus T3 hormone can stimulate the activity of neuropeptide Y neurons leading to inhibition of TRH production and thus demonstrating negative feedback loop, by subsequent decrease in TSH from anterior pituitary gland and downstream thyroid hormone from thyroid gland. The positive feedback mechanism is initiated on sensing low levels of peripheral concentration of thyroid hormones via increasing the TRH production from hypothalamus followed by rising TSH and thyroid hormone concentration in blood stream. ${ }^{19}$ The additional positive feedback sequence begins when the levels of thyroid hormones specifically the T4 levels are low resulting in increased number of TRH receptors and in TSH synthesis. This in turn leads to increased TSH response to TRH with subsequent increase in synthesis of thyroid hormone from thyroid gland. The exact mechanism follows the reverse path when the thyroid hormones levels are higher in peripheral blood representing an alternate negative feedback pathway. ${ }^{20}$

Thyroid hormone regulates normal growth and development via important metabolic pathways in children and adult as well. It also has a role in energy storage and expenditure for maintain energy balance by regulating metabolic processes in the skeletal muscle, brown fat, white fat, brain, pancreas, and liver. Thyroid hormone decreases hepatic gluconeogenesis by reducing insulin sensitivity in liver. It also has a key role in regulating processes involve in carbohydrate and cholesterol metabolic pathways by regulating gene expression and cross-talk with other nuclear receptors, such as liver $\mathrm{X}$ receptor, peroxisome proliferator-activated receptor and receptors involved in bile acid signaling pathways..$^{21}$ In brain, T4 hormone after converting in to active $\mathrm{T} 3$ can stimulate the activity of agouti-related protein (AgRP) neurons in arcuate nucleus, which in turn leads to increase in appetite. Agouti-related protein neuron stimulates proopiomelanocortin/ cocaine-amphetamine-regulated transcript neurons present in the arcuate nucleus to inhibit $\alpha$-melanocyte-stimulating hormone secretion leading to increase in appetite. Therefore under fasting conditions bioactive $\mathrm{T} 3$ hormone can promote increased feeding activity along with decrease in peripheral energy expenditure by its stimulatory effect on appetite and inhibitory action on TRH. Under normal circumstances, melanocyte-stimulating hormone has anorexigenic effect by decreasing the appetite. The decrease appetite leading to reduction in food intake will increase TRH secretion by stimulating TRH neurons, and consequently increasing thyroid hormone levels in the circulation. The thyroid hormones in blood stream will further lead to increase in peripheral energy expenditure and maintaining homeostasis in human physiology. ${ }^{19}$

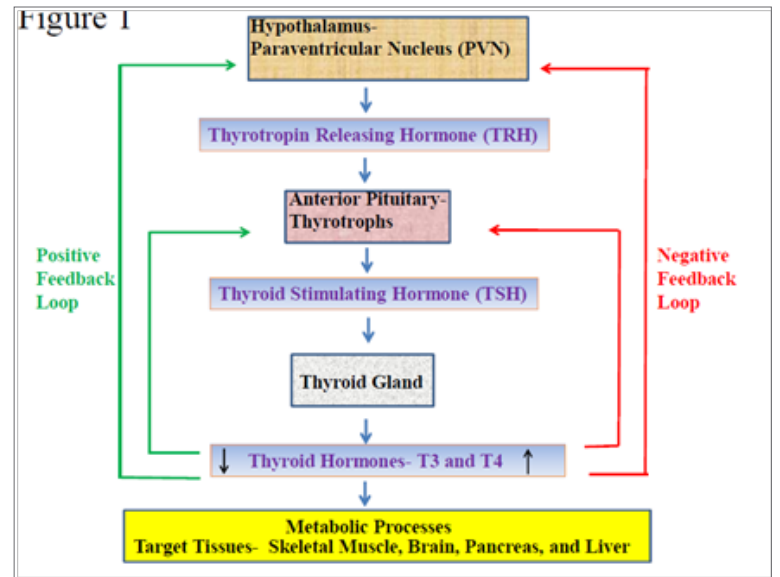

Figure I Flow-chart Illustrating Hypothalamo-Pituitary-Thyroid Axis and Feedback Loops: The hypothalamo-pituitary-thyroid axis begins from paraventricular nucleus (PVN) of hypothalamus producing thyrotropin releasing hormone (TRH) that causes thyrotrophs from anterior pituitary to release thyroid stimulating hormone (TSH) followed by secretion of thyroid hormones (T3 and T4) from thyroid gland. The positive feedback loop is initiated with decrease blood levels of thyroid hormones that results in increased production of TRH from hypothalamus and subsequent increase in TSH from anterior pituitary and thyroid hormones from thyroid gland. The negative feedback loop is initiated with increase blood levels of thyroid hormones which results in decreased production of TRH from hypothalamus and subsequent decrease in TSH from anterior pituitary and thyroid hormones from thyroid gland. Thyroid hormones (T3 and T4) upon releasing in the blood stream from thyroid gland regulate metabolic process by targeting several organs and tissues such as skeletal muscle, brain, pancreas, and liver.

\section{Case report}

During routine dissection of a female cadaveric body, ectopic thyroid tissue was found in the midline adjacent to the hyoid bone. The ectopic structure was oval in shape and measured $2.2 \times 1.6 \mathrm{x}$ $1.0 \mathrm{~cm}$ (Figure 2) (Figure 3). The inferior edge of the ectopic gland measured $10.2 \mathrm{~cm}$ from the superior margin of the thyroid isthmus. A thyroid gland of normal size and location was also present. The right lobe measured $5.0 \mathrm{~cm} \times 3.2 \mathrm{~cm} \times 3.4 \mathrm{~cm}$ and the left lobe measured $4.0 \mathrm{~cm} \times 2.5 \mathrm{~cm} \times 2.8 \mathrm{~cm}$ (Figure 2) (Figure 4). The isthmus was unusually wide, measuring $3.7 \mathrm{~cm}$ superior to inferior. There was no evidence of a pyramidal lobe. The external surface was red-brown with a normally lobulated appearance that matched that of the ectopic gland. The serial sectioning demonstrated normal fibrous septa that 
divided the gland into lobules measuring 1 to $2 \mathrm{~cm}$ in diameter (Figure 5). The appearance of the glandular parenchyma was unremarkable with a solid homogeneous red-brown cut surface with no nodules suggestive of goiter and no gross evidence of neoplasia.

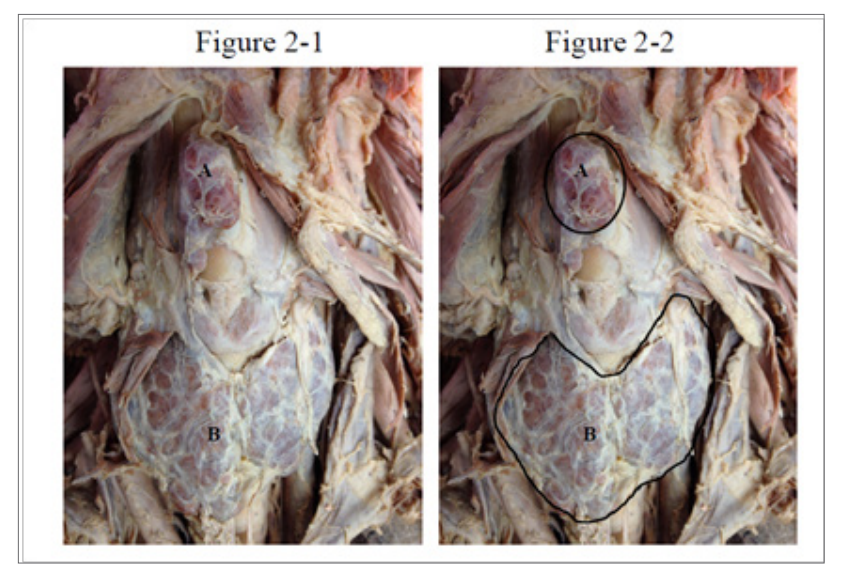

Figure 2 Thyroid Gland along with Ectopic Thyroid Tissue (AnteriorView);A) Ectopic Thyroid Tissue, B) Thyroid Gland.

Figure 2-I Depicts actual image from cadaver showing thyroid gland and ectopic thyroid tissue.

Figure 2-2 Depicts actual image from cadaver showing thyroid gland and ectopic thyroid tissue highlighted with border markings for easy identification from surrounding tissue.

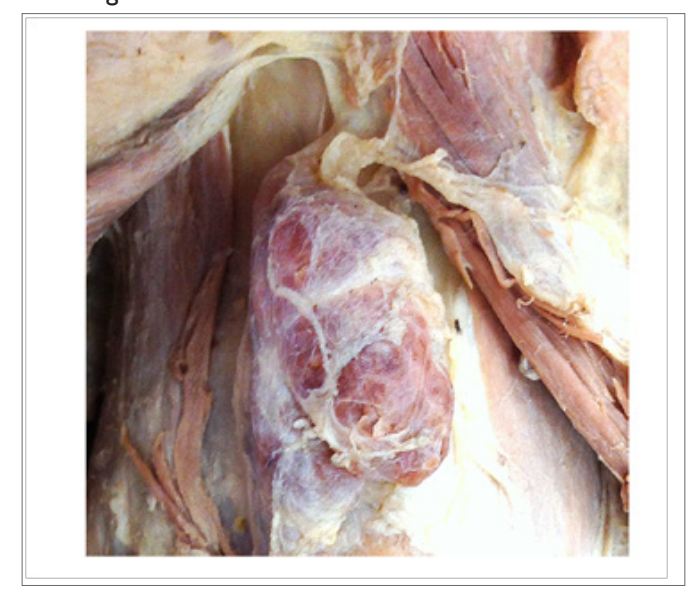

Figure 3 Ectopic Thyroid Tissues (AnteriorView).

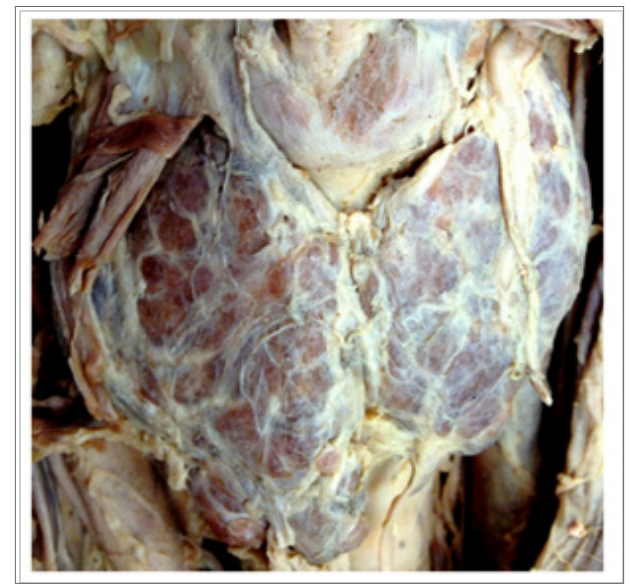

Figure 4 Thyroid Gland (Anterior View).

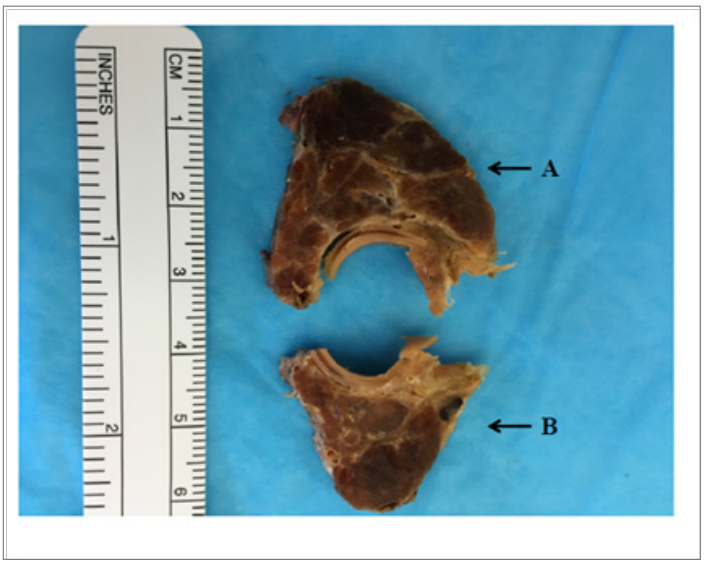

Figure 5 Serial Sectioning of Thyroid Gland;A) Right lobe, B) Left Lobe.

\section{Clinical perspective}

Most of the individuals with ectopic thyroid tissue are asymptomatic. However, ectopic thyroid tissue can present with similar pathology as the thyroid gland with hypo or hyperthyroidism including benign or malignant transformation. The ectopic thyroid tissue can also demonstrate other abnormalities like calcification, benign hyperplasia and multinodular goiter. More often ectopic thyroid tissue present with symptoms depending on its setting such as size of ectopic tissue, anatomical location, and the effect it has on the adjacent tissue. Patients with lingual thyroid most often presents with hypothyroid along with dysphagia, dysphonia, sensation of foreign body, cough, snoring, and sleep apnea with respiratory obstruction. The individuals with thyroglossal duct cyst or a submandibular thyroid are usually euthyroid and asymptomatic with palpable, painless, mobile, mass. Patients with ectopic thyroid located in the trachea are euthyroid but have other symptoms such as difficulty in swallowing, cough or hemoptysis, and dyspnea with or without stridor. Ectopic thyroid at the carotid bifurcation may present as a carotid body tumor and in some instance could be the only functioning thyroid tissue present in such individuals. ${ }^{14}$ Furthermore, ectopic thyroid tissue situated in the intrathoracic region like lung, heart, and mediastinum can have varied symptomatology and depending on its specific location, it can vary from asymptomatic to dry cough, dyspnea, hemoptysis and can even present with cardiac murmurs. However, more often such individual remains euthyroid in their thyroid functioning. ${ }^{5}$

Ectopic thyroid tissue in the regions like mammary glands, duodenum, intestine, spleen, liver, and pancreas can be detected incidentally with normal thyroid hormone levels on thyroid function test or rarely could be diagnosed as a cause of an abdominal pain. Patients with thyroid tissue in adrenal gland can present with hypertensive disorder. ${ }^{5,6}$ Other rare but an important location where thyroid tissue can be present is in ovary in the form of struma ovarii. The struma ovarii can be either euthyroid, or hyperthyroid in 5 to $15 \%$ patient population and present with no symptoms or lower abdominal pain with palpable lower abdominal mass, and abnormal vaginal bleeding. In very rare instances, patients with ectopy thyroid tissue in two different locations such as near tongue and hyoid regions can present with midline neck swelling and such dual ectopic thyroid patients usually are hypothyroid or be euthyroid with no symptoms at all. The normal physiological conditions such as puberty and pregnancy where there is high demand in thyroid hormones can cause increased production of thyrotropin leading to enlargement of the ectopic thyroid tissue. This increase in thyroid tissue could also be due 
to increase in levels of epidermal growth factor and human chorionic gonadotropin coupled with iodine deficiency in these patients. Pubertal and pregnancy changes leading to excessive enlargement of ectopic thyroid can present with pressure symptoms due to its encroachment on the surrounding tissue. ${ }^{6}$ There are also several reports demonstrating papillary carcinomas or a combination of follicular, papillary, and medullary carcinomas in ectopic thyroid tissue present in regions such as foramen cecum of tongue, thyroglossal duct, mediastinum, and ovaries. The incidence of malignancy arising in a lingual thyroid is estimated to be around $1 \% .^{7}$ The follicular carcinoma is the predominant form of malignancy seen in lingual thyroid as oppose to papillary carcinoma, which is the primary form of malignancy in thyroid gland seen predominantly in female patients. ${ }^{6}$ Lateral ectopic thyroid mass near sternocleidomastoid muscle and the common carotid artery has shown evidence of anaplastic carcinoma in the past. Papillary carcinoma has also been described in individuals with ectopic thyroid tissue present at dual location. In general malignant transformation in ectopic thyroid tissue giving rise to primary thyroid carcinomas are rare and such cases of primary thyroid carcinomas are usually diagnosed at the time of surgical excision of the tumor mass. However, along with having primary malignancy foci, ectopic thyroid tissue could also be the site for metastatic tumor. ${ }^{8}$

Radionuclide scans such as thyroid scintigraphy using nuclides of iodine and technetium like I-131, I-123 and Tc-99m are one of the most important diagnostic modalities to identify ectopic thyroid tissue due to the characteristics feature of thyroid tissue to concentrate iodine. Radiological imaging such as grayscale or color Doppler ultrasonography (USG), computed tomography (CT), and magnetic resonance imaging (MRI) also help in determining location and extent of the ectopic tissue. Thyroid function tests determining levels of T3, T4, TSH and thyroglobulin levels in the serum can be valuable tool in detecting ectopic thyroid tissue. Moreover, diagnostic procedure like fine needle aspiration (FNA) cytology can also be a useful diagnostic technique that may help identify thyroid as the tissue type of an abnormal mass and that may also help to differentiate between a benign and a malignant lesion. Positron emission tomography (PET) using fluor-18-deoxy-glucose (18F-FDG) is another such nuclear imaging technique that in recent years has emerged as a key diagnostic method in investigating differentiated thyroid carcinoma. Apart from these major investigations, there are some organ specific diagnostic modalities that can be utilized to rule out the presence of ectopic thyroid tissue in unusual locations namely echocardiography and coronary angiography for intracardiac thyroid tissue or barium swallow for locating thyroid tissue in gastrointestinal tract. ${ }^{6,8,22-24}$

Management of symptomatic ectopic thyroid has several treatment options including surgical excision of thyroid tissue using recent advances in minimal invasive techniques and/or radioiodine ablation procedure. Therefore, having comprehensive knowledge of morphological variations in the thyroid gland including presence of ectopic thyroid tissue would be very beneficial for clinicians evaluating results from thyroid scans and also while performing surgical procedures such as total, subtotal, and partial thyroidectomy. . $^{1,6,8}$

\section{Discussion}

The ectopic thyroid occurs due to embryonic malformation when thyroid fails to migrates from the region of thyroid anlage leaving behind the foremen cecum to its definitive location in front of the trachea. So due to this misplacement, the ectopic thyroid tissue can be found in any location along the path of migration from the foramen cecum to its normal location in front of the trachea and can even be seen in the abnormal location such as esophagus, lung, heart, aorta, mediastinum, duodenum, pancreas, gallbladder, small intestine, mesentery, adrenals, and ovaries. The ectopic thyroid tissue in ovaries, struma ovarii comprises of $2-4 \%$ of all ovarian teratoma and $1 \%$ of all ovarian tumors. However, most common location for the ectopic thyroid to be found is at the base of the foramen cecum as a mass in the dorsum of the tongue referred as lingual thyroid. In few instances thyroid tissue can develop in the submandibular region which could be due to defect in migration and fusion of lateral thyroid anlage with the median component of the thyroid gland or due to abnormal migration of the median thyroid anlage during embryonic development resulting in lateral thyroid mass. Although it is very rare, but there has been reports of two or three ectopic thyroids present in one individual. In dual ectopic cases the one of the location is usually around tongue and other site could be either above or below the hyoid bone. In reports describing triple ectopic, the thyroid tissues were present in three distinct sites on the lateral part of the neck region. ${ }^{2,6,8}$

Ectopic thyroid constitute most commonly occurring form of thyroid dysgenesis that accounts for 48-61\% of all forms of thyroid dysgenesis patients. Researchers have found several genes such as Transcription factor genes- (TITF1, NKX2-1, NKX2-3, NKX2-5, NKX2-6, PAX8, FOXE1, FOXA2, and HHEX), G protein coupled receptor gene- (TSHR), Tyrosine kinase receptor gene- (FFGR2), and Membrane protein gene- (NIS), that plays an important role during thyroid morphogenesis. The exact genetic mutation that causes ectopic thyroid is still not known completely, but research have shown that deleting FOXE1 gene in mouse can show abnormal migration leading to a phenotype that expresses thyroid ectopy. The reports describing discordance for thyroid dysgenesis in 12 of 13 monozygotic twin pairs shows that ectopic thyroid is not a hereditary transmitted disorder and that epigenetic processes may play a key role in the abnormal organogenesis of the thyroid. These findings suggest that genetic mutation that primarily occur after zygote formation lead to abnormal thyroid development. Moreover, it is not uncommon to notice ectopic thyroid along with other congenital anomalies especially cardiac defects. Also patients affected by mutation in these above mentioned genes shows varied phenotypic presentation. This suggests that many genes may be responsible for these abnormalities. Researchers have also shown through molecular research involving studies of structural variants, DNA methylation and transcriptome analysis showed that several genes are associated with certain signaling pathways in ectopic thyroid formation. These genes includes GPNMB, COL1A1, TBX1, LYZ, TNFAIP2, FGL2, PLCXD1, ENO3, DUSP6, BGN, ABCAI3, COL3A1, PBX4, CPEB4, TYROBP, and TXNIP, which are also involved in embryonic development. Moreover, genes such as SFRP2 and FRZB associated with Wnt pathway and gene MKRN1 associated with Wnt/Beta-Catenin pathway including non-canonical Wnt pathway have been studied for their embryogenic role in cell migration and thyroid development. It has also been shown that inhibition of Wnt pathway can decrease malignant process in thyroid carcinoma. Therefore future research is needed to define the exact molecular mechanism responsible for thyroid dysgenesis, which could provide a path for early diagnosis and intervention. ${ }^{2,6,8,25}$

\section{Conclusion}

In summary, ectopic thyroid tissue is a sporadic developmental anomaly that is resulted due to abnormal organogenesis during embryonic period. The ectopic thyroid tissue is referred as the thyroid present in any abnormal location other than its normal C5-T1 location 
in anterior lower neck region. ${ }^{2,3}$ The incidence of this rare entity is not known definitely but has shown high female predominance. ${ }^{26}$ The pathophysiology behind thyroid ectopy is not clearly illustrated but certain epigenetic and genetic mechanisms have been implicated in the thyroid dysgenesis resulting in to ectopic thyroid. ${ }^{2,6,8}$

Clinically, patients are often asymptomatic, but an ectopic gland can undergo any pathologic change that can occur in a normally located thyroid gland manifesting in to hypothyroidism and hyperthyroidism including malignancy. ${ }^{5,6,14}$ Imaging modalities such as USG, CT, MRI, and PET are useful techniques to locate ectopic thyroid. However, thyroid scintigraphy is most important radiological test to diagnose ectopic thyroid tissue. ${ }^{6,8,22-24}$ Treatment procedures such as surgical excision and radioiodine ablation both has role in managing symptomatic ectopic thyroid. ${ }^{1,6,8}$

Thus, in this report we have presented a photographic evidence of ectopic thyroid gland and normally located thyroid gland the anterior neck in a female cadaver along with a brief review summarizing important facets of normally located thyroid gland and ectopic thyroid that could be beneficial in understanding such rare but important congenital abnormality. In turn this could result in newer and better strategies in diagnosing and managing such rare thyroid anomaly. Moreover, the report also highlights certain key intricate aspects of organogenesis that have significance in conducting basic as well as clinical research involving ectopic thyroid tissue.

\section{Acknowledgments}

None.

\section{Conflict of interests}

The authors have no conflict of interests.

\section{References}

1. Rajkonwar AJ, Kusre G. Morphological Variations of the Thyroid Gland among the People of Upper Assam Region of Northeast India: A Cadaveric Study. J Clin Diagn Res. 2016;10(12):AC01-AC03.

2. De Felice M, Di Lauro R. Thyroid development and its disorders: genetics and molecular mechanisms. Endocr Rev. 2004;25(5):722-746.

3. Standring S. Gray's Anatomy: The Anatomical Basis of Clinical Practice, 40th edn. Churchill Livingstone Elsevier, Amsterdam; 2008.

4. Hollander EJ, Visser MJ, van Baalen JM. Accessory thyroid gland at carotid bifurcation presenting as a carotid body tumor: case report and review of the literature. J Vasc Surg. 2004;39(1):260-262.

5. Bhatnagar KP, Nettleton GS, Wagner CE. Subisthmic accessory thyroid gland in man: a case report and a review of thyroid anomalies. Clin Anat. 1997; 10:341-344.

6. Ibrahim NA, Fadeyibi IO. Ectopic thyroid: etiology, pathology and management. Hormones (Athens). 2011;10(4):261-269.

7. Kousta E, Konstantinidis K, Michalakis C, et al. Ectopic thyroid tissue in the lower neck with a coexisting normally located multinodular goiter and brief literature review. Hormones (Athens) 2005;4(4):231-234.
8. Noussios G, Anagnostis P, Goulis DG, et al. Ectopic thyroid tissue: anatomical, clinical, and surgical implications of a rare entity. Eur J Endocrinol. 2011;165(3):375-382.

9. Bhargav PR. Salient anatomical landmarks of thyroid and their practical significance in thyroid surgery: a pictorial review of thyroid surgical anatomy (revisited). Indian J Surg. 2014;76(3):207-211.

10. Maenhaut C, Christophe D, Vassart G, et al. Ontogeny, Anatomy, Metabolism and Physiology of the Thyroid. Endotext [Internet]. 2015.

11. Pawlina W, Ross M, Histology. A Text and Atlas with Correlated Cell and Molecular Biology, seventh edition. Wolters Kluwer Health, Philadelphia, 2016. pp. 757-764.

12. Costache A, Dumitru M, Anghel I, et al. Ultrasonographic anatomy of head and neck--a pictorial for the ENT specialist. Med Ultrason 2015;17:104108.

13. Milojevic B, Tosevski J, Milisavljevic M, et al. Pyramidal lobe of the human thyroid gland: an anatomical study with clinical implications. Rom J Morphol Embryol. 2013;54(2):285-289.

14. Ranade AV, Rai R, Pai MM, et al. Anatomical variations of the thyroid gland: possible surgical implications. Singapore Med J. 2008;49(10):831834.

15. Gervasio A, Mujahed I, Biasio A, et al. Ultrasound anatomy of the neck: The infrahyoid region. J Ultrasound. 2010;13(3):85-89.

16. Brent GA. Mechanisms of thyroid hormone action. J Clin Invest. 2012;122(9):3035-3043.

17. Ganong WF. The thyroid gland. In: Review of medical physiology, 22nd edition, New York; McGraw-Hill, 2005. p. 317-332.

18. Krukowski ZH. The thyroid and the thyroglossal tract. In: Russell RCG, et al. editors). Bailey and Love's short practice of surgery 24th edition, London; Arnold, 2004. pp. 776-803.

19. Herwig A, Ross AW, Nilaweera KN, et al. Hypothalamicthyroid hormone in energy balance regulation. Obes Facts. 2008;1(2):71-79.

20. Nussey S, Whitehead S. Endocrinology: An Integrated Approach. Oxford:BIOS Scientific Publishers; Chapter 3, The thyroid gland. 2001.

21. Mullur R, Liu YY, Brent GA. Thyroid hormone regulation of metabolism. Physiol Rev. 2014;94(2):355-382.

22. Czepczyński R. Nuclear medicine in the diagnosis of benign thyroid diseases. Nucl Med Rev Cent East Eur. 2012;15(2):113-119.

23. Oguz A, Tuzun D, Ozdemir E, et al. Importance of ectopic thyroid tissue detected in the midline of the neck: single center experience. Arch Endocrinol Metab. 2015;60:231-235.

24. Salvatori M, Biondi B, Rufini V. Imaging in endocrinology: 2-[18F]-fluoro2-deoxy-D-glucose positron emission tomography/computed tomography indifferentiated thyroid carcinoma: clinical indications and controversies indiagnosis and follow-up. Eur J Endocrinol. 2015;173(3):R115-R130.

25. Abu-Khudir R, Paquette J, Lefort A, et al. Transcriptome, methylome and genomic variations analysis of ectopic thyroid glands. PLoS One. 2010;5(10):e13420.

26. Yoon JS, Won KC, Cho IH, et al. Clinical characteristics of ectopic thyroid in Korea. Thyroid. 2007;17(11):1117-1121. 